\title{
Sclerostin: a novel role in TNF arthritis?
}

Researchers studying the role of sclerostin in mouse models of inflammatory arthritis have been surprised to find that sclerostin blockade can aggravate TNF-driven inflammatory joint destruction. This finding suggests that sclerostinblocking antibodies (currently under investigation as a treatment for osteoporosis) could potentially worsen bone erosion in patients with rheumatoid arthritis (RA). "Anti-sclerostin antibody treatment [could] be contraindicated in a subset of RA patients or in patients with chronic TNF-dependent comorbidities," warns Thomas Pap, corresponding author of the study.

The group's finding that treatment with sclerostin-blocking antibodies led to profound bone loss in mice engineered to constitutively overexpress human TNF (hTNFtg mice, which develop an RA-like destructive arthritis) came as a surprise because Sost ${ }^{-/}$mice (which lack sclerostin) have a high bone mass phenotype driven by upregulation of both Wnt- $\beta$-catenin signalling and osteoblast-specific gene expression. As inhibition of Wnt- $\beta$-catenin signalling (leading to suppression of bone formation) is a key feature of RA, the researchers were expecting to find that blocking sclerostin would ameliorate arthritis in this model.
4

...sclerostin-

blocking antibodies

... could

potentially worsen bone

erosion in patients with rheumatoid arthritis 5
In further experiments, the researchers showed that in mice with partially TNF-dependent arthritis, resulting from immunization with glucose-6-phosphate isomerase (G6PI), sclerostin inhibition neither worsened nor ameliorated focal erosions and inflammation in diseased joints, suggesting that different repair mechanisms are activated in response to systemic and local bone loss. By contrast, sclerostin inhibition did ameliorate disease in the $\mathrm{K} / \mathrm{B} \times \mathrm{N}$ serum transfer mouse model of RA, in which arthritis is IL-1-driven and TNF-receptor-independent. Taken together, these observations suggest that the extent to which TNF is involved in the pathogenesis of arthritis influences whether sclerostin blockade will have a protective or erosionintensifying effect on bone. "This study has immediate impact on current clinical trials using anti-sclerostin antibodies,"
However, as previously published work had shown healing of bone erosions in hTNFtg mice treated with both anti-TNF agents and sclerostin-blocking antibodies, the researchers consider that the adverse effects of sclerostin blockade on bone might be avoided if the inflammatory effects of TNF are adequately suppressed before initiating anti-sclerostin therapy.

The team also found that sclerostin expression is not restricted to osteocytes, as previously thought: large amounts of sclerostin were released by fibroblast-like synoviocytes obtained from patients with rheumatoid arthritis (RA) in response to TNF exposure. Moreover, they showed that sclerostin requires LDL-receptor related protein 6 (LRP6) to block the TNF-induced activation of nuclear factor $\kappa \mathrm{B}$ (NFkB) and p38 - a key step in the development of arthritis. Thus, in chronic TNF-mediated inflammation, sclerostin might be upregulated as part of an attempt to re-establish bone homeostasis.

As Pap sums up, "[Our findings point] to a previously unrealized protective role of sclerostin in TNF-mediated chronic inflammation."

Caroline Barranco

ORIGINAL ARTICLE Wehmeyer, C. et al. Sclerostin inhibition promotes TNF-dependent inflammatory joint destruction. Sci. Transl. Med. 8 330Ra35 (2016)

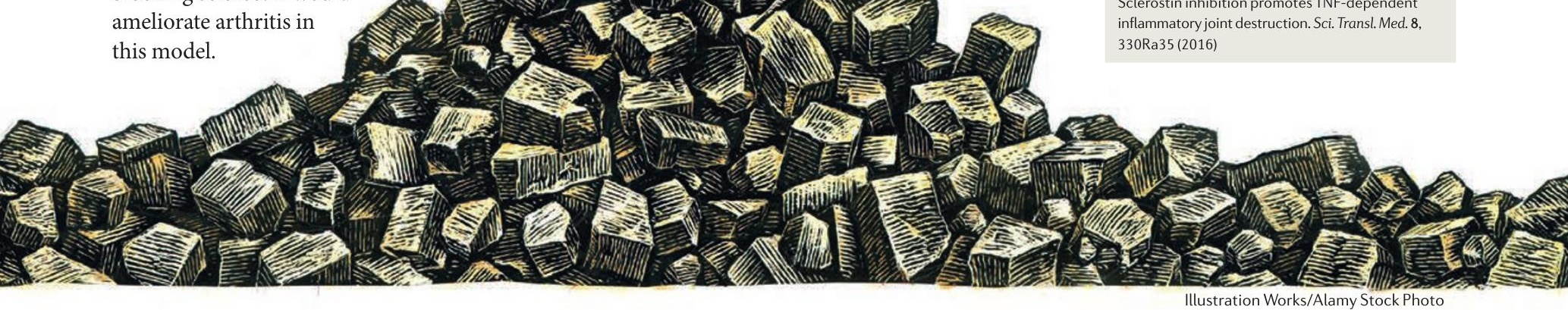

